\title{
Clinical importance of chemokines and inflammatory cytokines for patient care following percutaneous nephrolithotripsy
}

\author{
JUNRONG WANG $^{1}$, WEI WANG ${ }^{1}$, WEI GUO ${ }^{2}$, YANRU MA ${ }^{1}$, TIANHUI $\mathrm{JI}^{1}$ and BAODI ZHANG ${ }^{3}$ \\ ${ }^{1}$ Operating Room, First Branch of Mudanjiang Medical University Affiliated Hongqi Hospital; \\ ${ }^{2}$ Department of Opthalmology, Mudanjiang Medical University Affiliated Hongqi Hospital; \\ ${ }^{3}$ Department of Surgery, First Branch of Mudanjiang Medical University Affiliated Hongqi Hospital, \\ Mudanjiang, Heilongjiang 157011, P.R. China
}

Received February 14, 2017; Accepted November 17, 2017

DOI: $10.3892 /$ etm.2017.5645

\begin{abstract}
Chemokines are a class of proteins with low molecular weight that serve important roles in the progression of inflammation. Percutaneous nephrolithotripsy is a surgical technique in which lasers or ultrasound are utilized to break down and/or remove kidney stones. In order to ensure a full recovery following surgery, effective patient care and nursing are required. In the present study, a total of 348 patients with kidney stones were recruited and the clinical importance of chemokines and inflammatory cytokines for the nursing of patients during perioperative period was investigated. Plasma levels of inflammatory cytokines, as well as chemokines in the $\mathrm{C}, \mathrm{CC}$ and $\mathrm{CXC}$ families, were analyzed in patients following percutaneous nephrolithotripsy. Correlations between chemokines and inflammatory cytokines and the urinary concentration of calcium oxalate were also investigated. The results indicated that plasma levels of $\mathrm{C}$ and $\mathrm{CC}$ chemokines were downregulated in patients following percutaneous nephrolithotripsy, whereas the plasma concentrations of CXC chemokines were upregulated. Plasma concentration levels of inflammatory cytokines interleukin (IL)-8, IL-1, IL-17 and tumor necrosis factor (TNF)- $\alpha$ were significantly downregulated in patients following percutaneous nephrolithotripsy; however, no significant differences were observed in plasma levels of IL-6 and IL-10 pre- and post-surgery. Regression analysis revealed that plasma concentration levels of chemokine $\mathrm{C}$ motif ligand, which is a $\mathrm{C}$ chemokine, chemokine ligand 2, which is a CC chemokine, and TNF- $\alpha$ were positively correlated with the urinary concentration of calcium oxalate during the perioperative period. The results of the present study indicate that plasma levels of chemokines and
\end{abstract}

Correspondence to: Professor Baodi Zhang, Department of Surgery, First Branch of Mudanjiang Medical University Affiliated Hongqi Hospital, 2 Taiping Road, Xian, Mudanjiang, Heilongjiang 157011, P.R. China

E-mail: zhangbaodimdj@163.com

Key words: chemokine, inflammatory cytokines, nursing, percutaneous nephrolithotripsy inflammatory cytokines are clinically important for nursing of patients who experienced percutaneous nephrolithotripsy.

\section{Introduction}

Kidney stones are a common problem affecting the urinary system that significantly affect metabolism and impede physical activity (1). Studies investigating the causes of disease have reported that several factors contribute to the formation of stones, including age, sex, ethnicity, genetic and environmental factors, dietary habits, occupation, metabolic abnormalities, urinary tract obstruction, infection and drug use $(2,3)$. Clinical pathology has demonstrated that calcium oxalate is the primary constituent of kidney stones (4). Percutaneous nephrolithotripsy is a commonly used and effective surgical treatment for solitary kidney stones $(5,6)$. Laparoscopy-assisted transmesocolonic percutaneous nephrolithotripsy is considered to be a safe and effective treatment option in cases of ectopic kidney stone disease (7). Clinical care is important for recovery from percutaneous nephrolithotripsy in clinical patients (8).

Chemokines are a class of low molecular weight proteins that attract white blood cells to infection sites and serve an important role in immunological surveillance $(9,10)$. Previous studies have suggested that chemokine levels may be used as an indicator of the progression of inflammation, with immune cell migration increasing with the concentration of chemokines $(11,12)$. Changes in the plasma concentration of chemokines are associated with several diseases, including netherton syndrome and cancer and are regarded as prognostic indicators $(13,14)$. In recent years, the mechanism by which chemokines mediate systemic inflammation has been investigated and analyzed; however, the clinical importance of chemokines for the postoperative care of patients who have undergone percutaneous nephrolithotripsy has not been studied (15).

Inflammation is commonly observed in patients following percutaneous nephrolithotripsy (16). It has previously been reported that predictors, including sepsis and fever were regulated in the systemic inflammatory response following percutaneous nephrolithotripsy and patients with the systemic inflammatory response syndrome risk factors must 
be aggressively treated to prevent postoperative sepsis (17). $\mathrm{Lu}$ et al (18) suggested that renal tubular epithelial cell injury, apoptosis and inflammation are associated with melamine-related kidney stone formation. These findings are important for developing an understanding of the pathogenesis of melamine-related kidney stone formation and estimating patient prognoses (18). The molecular mechanisms of crystal-associated kidney inflammation and injury have implications in cholesterol embolisms, crystalline nephropathies and kidney stone disease and may provide novel therapeutic options for progressive tissue remodeling in patients with kidney stone disease (19).

The aim of the present study was to investigate changes in the plasma levels of chemokines and inflammatory cytokines in patients undergoing percutaneous nephrolithotripsy pre- and post-treatment. The correlation between urinary concentrations of calcium oxalate and plasma levels of chemokines following percutaneous nephrolithotripsy was also investigated. The results of the present study demonstrated the clinical importance of chemokines and inflammatory cytokines for the clinical care of patients following percutaneous nephrolithotripsy.

\section{Materials and methods}

Ethics statement. The present study was approved by the Ethics Committee of the First Branch of Hongqi Hospital of Mudanjiang Medical University (Mudanjiang, China). This clinical investigation (trial no. HMCH2010072508) was performed in strict accordance with the Guide for Hongqi Hospital of Mudanjiang Medical University (20) between May 2010 and October 2015. A total of 348 patients with kidney stones were required to review trial protocols and amendments and provide written informed consent in the Hongqi Hospital of Mudanjiang Medical University. All patients were asked to provide $5 \mathrm{ml}$ venous blood in hospital and provided written informed consent.

Patient characteristics. A total of 348 (male/female, 263/86) patients with kidney stones were enrolled in the current study. The mean age was 34.6 years old (range, 22.6-40.6). Patients with chronic renal failure and diabetes mellitus were excluded from the current study. All patients were required to provide written informed consent.

ELISA. Plasma levels of chemokine C motif ligand (XCL)1 (cat no. DXCL10; Bio-Rad Laboratories, Inc., Hercules, CA, USA), XCL2 (cat no. CSB-EL026187HU; Cusabio Life Science, Wuhan, China), chemokine C-X3-C motif (CX3C; cat no. DY365) CX3C ligand 1 (CX3CL1; cat no. DCX310), chemokine C-C motif ligand (CCL)1 (cat no. DY272), CCL2 (cat no. DCP00), CCL3 (cat no. DMA00), CCL4 (cat no. DMB00), chemokine $\mathrm{C}-\mathrm{X}-\mathrm{C}$ motif ligand (CXCL)1 (cat no. DGR00B), CXCL2 (cat no. DY276-05), CXCL3 (cat no. RNC200), CXCL4 (cat no. DPF40), interleukin (IL)-8 (cat no. D8000C), IL-1 (cat no. DLB50), IL-17 (cat no. D1700), tumor necrosis factor (TNF)- $\alpha$ (cat no. DTA00C), IL-6 (cat no. D6050) and IL-10 (cat no. D1000B; all Bio-Rad Laboratories Inc.) were detected prior to and following percutaneous nephrolithotripsy using ELISA kits (Bio-Techne,
Minneapolis, MN, USA) according to the manufacturer's protocol. Patients' urinary concentrations of calcium oxalate were also assessed pre- and post-surgery using an ELISA kit (cat no. K663-100; Beijing Solarbio Science \& Technology Co., Ltd., Beijing, China).

Regression analysis. Peripheral venous blood $(5 \mathrm{ml})$ and urine samples $(5 \mathrm{ml})$ were collected 1 day prior to and 5 days following percutaneous nephrolithotripsy. Blood samples were treated with heparin (10 IU/ml; Sigma-Aldrich; Merck KGaA, Darmstadt, Germany). Plasma samples were prepared immediately by centrifugation $\left(2,000 \mathrm{x} \mathrm{g}, 4^{\circ} \mathrm{C}\right.$ for $\left.10 \mathrm{~min}\right)$ of peripheral venous blood. The plasma levels of XCL1, CCL2 and TNF- $\alpha$, as well as the urinary concentration of calcium oxalate were calculated from the ELISA results using regression analysis with least square convergence (21). The predicted curve that resulted in the lowest sum of squares was determined to be the best fit. If the fit is robust, the parameters of the observed curve may be inferred from those of the predicted.

Statistical analysis. Data are presented as the mean + or \pm standard error of the mean. All data were analyzed by SPSS 19.0 software (IBM Corp., Armonk, NY, USA). Statistical differences between groups were assessed using one-way analysis of variance from 6 replicate experiments with the post-hoc Dunnett's test. $\mathrm{P}<0.05$ was considered to indicate a statistically significant difference.

\section{Results}

Plasma C chemokine levels following percutaneous nephrolithotripsy. Plasma levels of the C chemokines XCL1, XCL2, CX3C and CX3CL1 were assessed in patients following percutaneous nephrolithotripsy. Plasma levels of XCL1 and XCL2 were significantly downregulated in patients following percutaneous nephrolithotripsy compared with pre-operative values $(\mathrm{P}<0.01$; Fig. $1 \mathrm{~A}$ and $\mathrm{B})$. Plasma levels of $\mathrm{CX} 3 \mathrm{C}(\mathrm{P}<0.05$; Fig. 1C) and CX3CL1 (P<0.01; Fig. 1D) were also significantly downregulated in patients following surgery compared with pre-operative values. These changes in the plasma levels of $\mathrm{C}$ chemokines may be used as postoperative diagnostic criteria for patients after percutaneous nephrolithotripsy.

Plasma CC chemokine levels following percutaneous nephrolithotripsy. The concentrations of CC chemokines CCL1, CCL2, CCL3 and CCL4 in the plasma of patients following percutaneous nephrolithotripsy were assessed. It was demonstrated that CCL1 and CCL2 levels were significantly downregulated following percutaneous nephrolithotripsy compared with pre-operative values $(\mathrm{P}<0.01$; Fig. $2 \mathrm{~A}$ and $\mathrm{B})$. CCL3 ( $\mathrm{P}<0.01$; Fig. 2C) and CCL4 ( $\mathrm{P}<0.05$; Fig. 2D) expression levels were also observed to be significantly decreased following percutaneous nephrolithotripsy compared with pre-operative values. These results suggest that the expression of CC chemokines in is downregulated in patients following percutaneous nephrolithotripsy.

Plasma CXC chemokine levels following percutaneous nephrolithotripsy. CXC chemokine expression following percutaneous nephrolithotripsy was assessed. Plasma levels of 

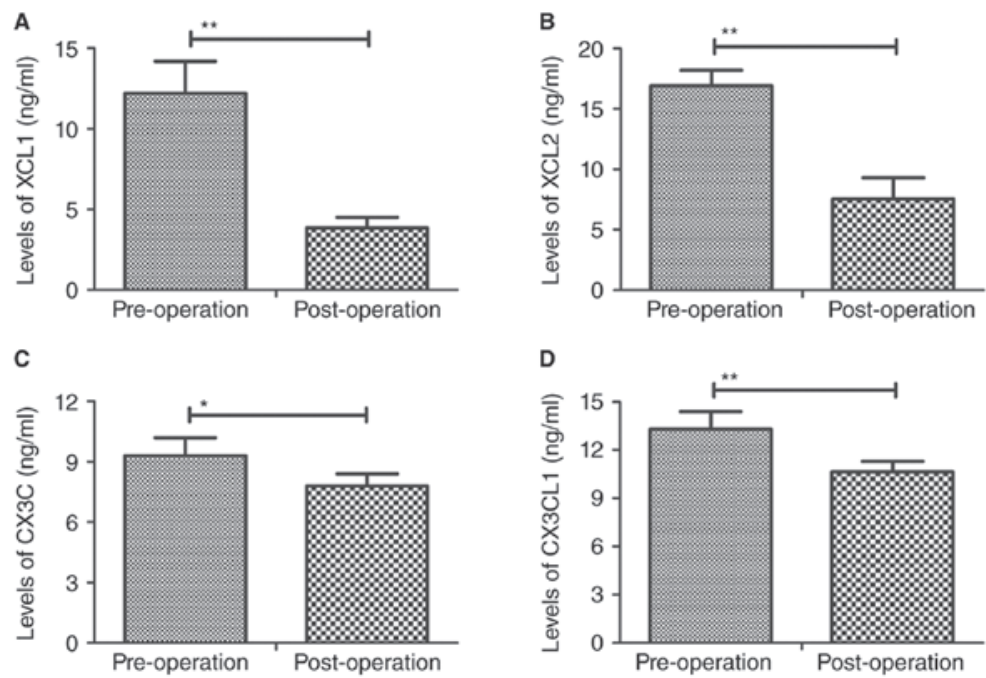

Figure 1. Plasma levels of $\mathrm{C}$ chemokines prior to and following percutaneous nephrolithotripsy. Plasma concentrations of (A) XCL1, (B) XCL2, (C) CX3C and (D) CX3CL1 were assessed using ELISA. Data are presented as the mean \pm standard error of the mean. " $\mathrm{P}<0.05$ and ${ }^{* *} \mathrm{P}<0.01$. XCL, chemokine $\mathrm{C}$ motif ligand; CXC3, chemokine C-X3-C motif; CXC3L1, CX3C ligand 1.
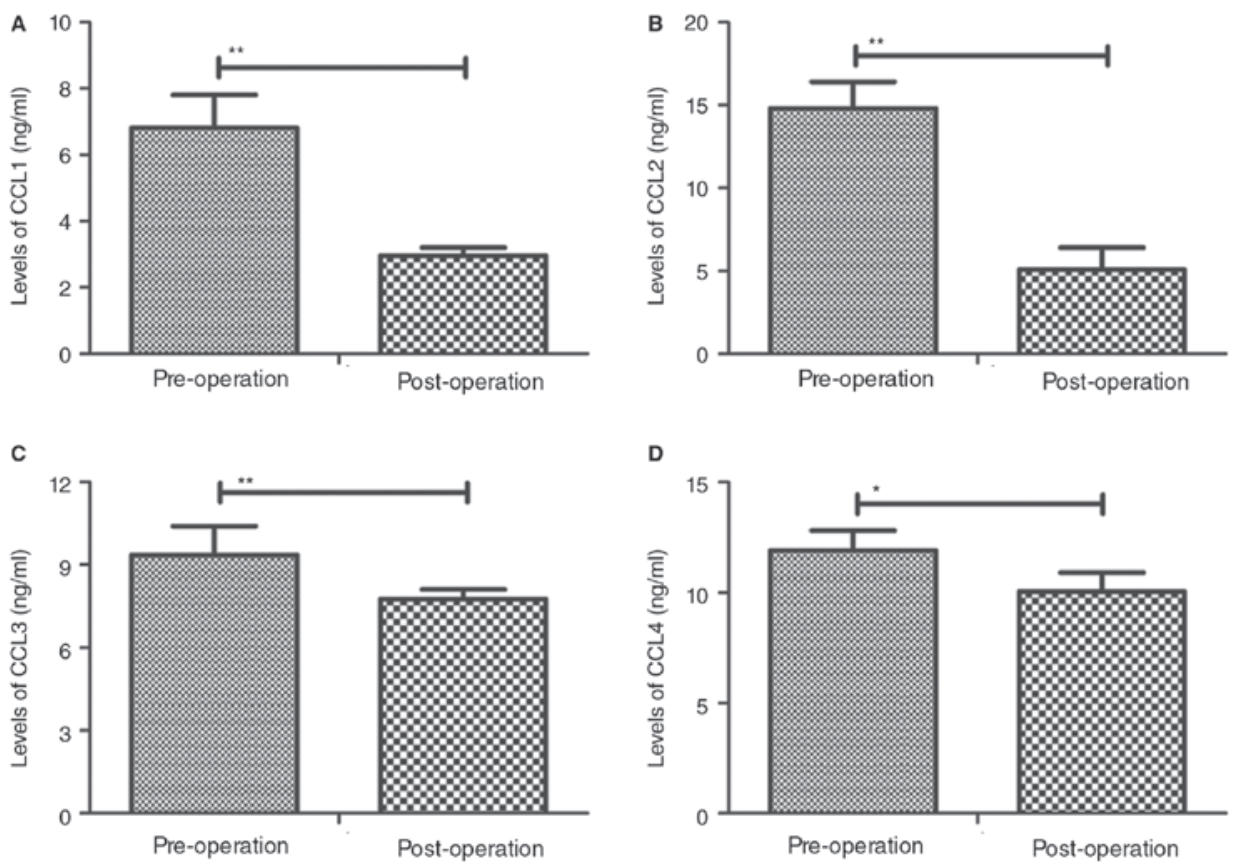

Figure 2. Plasma levels of CC chemokines prior to and following percutaneous nephrolithotripsy. Plasma concentrations of (A) CCL1, (B) CCL2, (C) CCL3 and (D) CCL4 were assessed using ELISA. Data are presented as the mean \pm standard error of the mean. "P $<0.05$ and ${ }^{* * *} \mathrm{P}<0.01$. CCL, chemokine $\mathrm{C}-\mathrm{C}$ motif ligand.

CXCL1 and CXCL2 were significantly upregulated in patients following percutaneous nephrolithotripsy compared with pre-operative values $(\mathrm{P}<0.01$; Fig. $3 \mathrm{~A}$ and $\mathrm{B})$. Furthermore, CXCL3 and CXCL4 expression levels were increased post-surgery compared with pre-operative values $(\mathrm{P}<0.05$; Fig. 3C and D).

Inflammatory cytokine levels following percutaneous nephrolithotripsy. The plasma levels of certain inflammatory cytokines were assessed in patients prior to and following percutaneous nephrolithotripsy. The results indicated that the concentrations of IL-8, IL-1, IL-17 and TNF- $\alpha$ were significantly downregulated in following percutaneous nephrolithotripsy compared with pre-operative values $(\mathrm{P}<0.01$; Fig. 4A-D). Conversely, the plasma levels of IL-6 and IL-10 were significantly upregulated in patients following percutaneous nephrolithotripsy compared with pre-operative values $(\mathrm{P}<0.01$; Fig. 4E and F). Collectively, these data suggest that the expression of inflammatory cytokines in the plasma is altered in patients following percutaneous nephrolithotripsy and that upregulating inflammation cytokines may optimize anti-inflammatory drug therapy.

Regression analysis for plasma levels chemokine and urinary concentration of calcium oxalate in patients after percutaneous nephrolithotripsy. Regression analysis was performed 

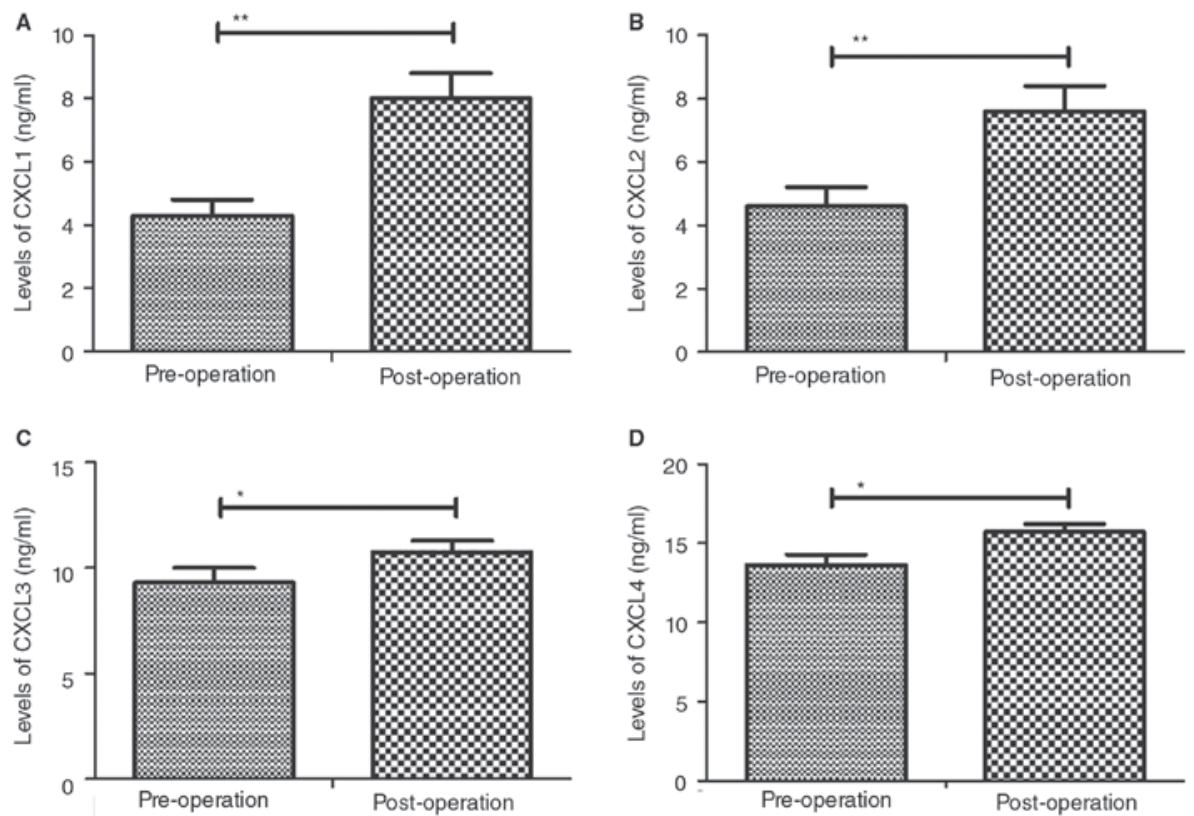

Figure 3. Plasma levels of CXC chemokines prior to and following percutaneous nephrolithotripsy. Plasma concentrations of (A) CXCL1, (B) CXCL2, (C) CXCL3 and (D) CXCL4 were assessed using ELISA. Data are presented as the mean \pm standard error of the mean. ${ }^{*}<0.05$ and ${ }^{* *} \mathrm{P}<0.01$. CXCL, chemokine C-X-C motif ligand.
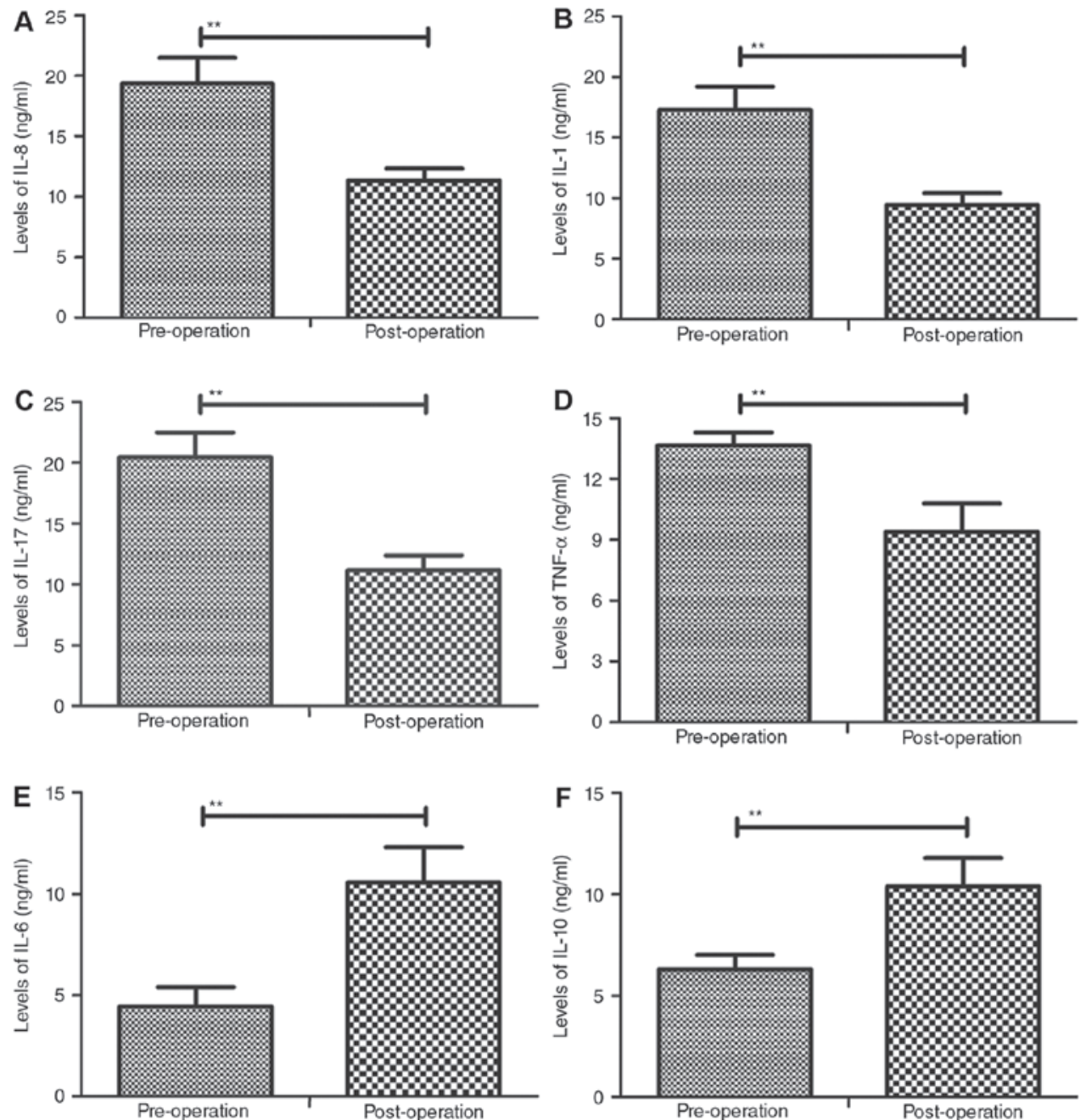

Figure 4. Plasma levels of inflammatory cytokines prior to and following percutaneous nephrolithotripsy. Plasma concentrations of (A) IL-8, (B) IL-1, (C) IL-17, (D) TNF- $\alpha$, (E) IL-6 and (F) IL-10 were assessed using ELISA. Data are presented as the mean \pm standard error of the mean. ${ }^{* *}$ P $<0.01$. IL, interleukin; TNF, tumor necrosis factor. 
A

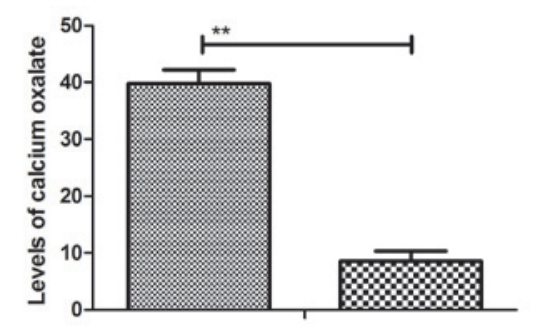

C

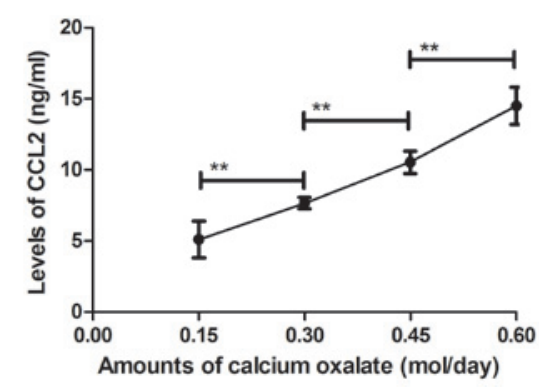

B

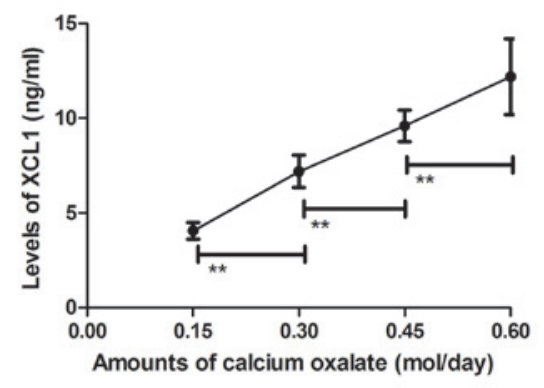

D

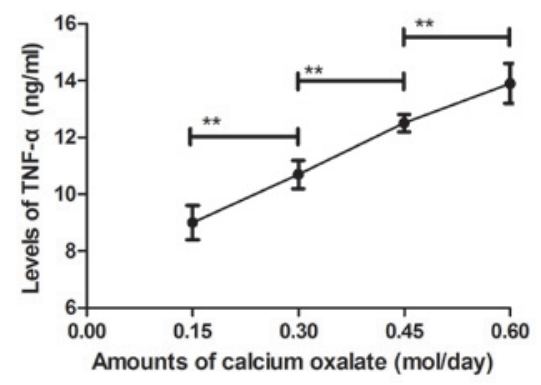

Figure 5. Regression analyses to assess the correlation between plasma cytokine levels and urinary concentrations of calcium oxalate following percutaneous nephrolithotripsy. (A) Urinary concentration of calcium prior to and following percutaneous nephrolithotripsy as assessed using ELISA. Plasma levels of (B) XCL1, (C) CCL2 and (D) TNF- $\alpha$ are positively correlated with urinary calcium oxalate following percutaneous nephrolithotripsy. ${ }^{* *}$ P $<0.01$. IL, interleukin; TNF, tumor necrosis factor.

for plasma levels of chemokines and urinary concentrations of calcium oxalate following percutaneous nephrolithotripsy. ELISA results revealed that the urinary concentration of calcium oxalate was downregulated in patients following percutaneous nephrolithotripsy compared with pre-operative values $(\mathrm{P}<0.01$; Fig. 5A). The results demonstrated that the $\mathrm{C}$ chemokine XCL1 and CC chemokine CCL2 were positively correlated with the urinary concentration of calcium oxalate following percutaneous nephrolithotripsy $(\mathrm{P}<0.01$; Fig. $5 \mathrm{~B}$ and $\mathrm{C}$ ). It was demonstrated that plasma levels of TNF- $\alpha$ are positively correlated with the urinary concentration of calcium oxalate in patients following percutaneous nephrolithotripsy $(\mathrm{P}<0.01$; Fig. 5D). Collectively, the results of regression analyses indicate that plasma chemokine levels are positively correlated with the urinary concentration of calcium oxalate in patients following percutaneous nephrolithotripsy.

\section{Discussion}

Percutaneous nephrolithotripsy is a delicate and highly technical clinical procedure used to treat patients with kidney stones $(22,23)$. It has previously been reported that postoperative medication is beneficial for the recovery of patients following percutaneous nephrolithotripsy (24). The expression of chemokines in glomeruli is correlated with nephrosis and C-C chemokine receptor 2 is associated with inflammatory responses in post-kidney transplant patients $(25,26)$. Furthermore, inflammatory cytokines are important post-surgical prognostic factors (27). In the present study, the clinical importance of chemokine and inflammatory cytokines for the nursing of patients following percutaneous nephrolithotripsy was investigated. The results revealed that plasma concentrations of $\mathrm{C}$ and
CC chemokines were downregulated, whereas CXC chemokines compared with pre-surgical values. The plasma levels of inflammatory cytokines IL-8, IL-1, IL-17 and TNF- $\alpha$ were downregulated, whereas IL-6 and IL-10 were upregulated in patients following percutaneous nephrolithotripsy. Regression analysis demonstrated that the expression of $\mathrm{C}$ and $\mathrm{CC}$ chemokines was positively correlated with the urinary concentration of calcium oxalate following percutaneous nephrolithotripsy. These results suggest that downregulation of chemokines and inflammatory cytokines are essential for the clinical nursing of patients following percutaneous nephrolithotripsy, which may contribute to postoperative recovery.

A previous study has reported that metamorphic folding of XCL1 is required for potent antimicrobial activity (28). In addition, Gombert et al (29) demonstrated that interactions between the recruitment of $\mathrm{T}$ cells and Langerhans-type dendritic cells occur in atopic skin inflammation. Upregulation of IL-6, IL-8 and CCL2 expression and the progression of tissue injury following acute inflammation has previously been investigated (30). The urinary concentration of calcium is associated with kidney stones and is typically used as a prognostic factor for patients who have undergone surgical intervention for kidney stones $(31,32)$.

The role of inflammation in diabetes and diabetic kidney disease is becoming more widely accepted than metabolic syndrome (33). Inflammatory responses caused by kidney stone may lead to other urinary system infections. It has been demonstrated that TNF- $\alpha$ is associated with a number of kidney diseases and the correlation with clinical variables has previously been reported in chronic kidney disease (34-36). Zhang et al (37) indicated that IL-1 inhibitor may mitigate liver and kidney dysfunction and improve 
survival in rat endotoxemia. Sirota et al (38) have demonstrated that plasma IL-8 is a biomarker of acute kidney injury. Vasanthakumar et al (39) suggested that IL-17 was positively and negatively correlated with fasting and postprandial glucose levels, respectively, and glycated hemoglobin (HbAlc) was observed in subjects with diabetic kidney disease. Ahuja et al (40) indicated that that circulating IL-6 mediates inflammatory responses via CXCL1 production following acute kidney injury in mice. Furthermore, the role of IL-10 has been reported in normal renal physiology, during acute kidney injury and in the development of chronic renal failure (41). The results demonstrated that plasma levels of IL-8, IL-1, IL-17 and TNF- $\alpha$ were downregulated; however, plasma concentration levels of IL- 6 and IL-10 were upregulated in patients after percutaneous nephrolithotripsy compared to pre-operation. Clinical research also indicated the importance of inflammation for nursing research (42). Regression analysis indicates that that plasma levels of TNF- $\alpha$ were positively correlated with urinary concentration of calcium oxalate in patients after percutaneous nephrolithotripsy. These results suggest that monitoring changes of plasma levels of inflammatory cytokines is clinical significant for nursing of patients after percutaneous nephrolithotripsy.

In conclusion, this study is the first to report the clinical importance of chemokine and inflammatory cytokines for the nursing of patients who have undergone percutaneous nephrolithotripsy. The correlation between plasma concentration levels of chemokines and inflammation cytokines and the urinary concentration of calcium oxalate in patients was also assessed. These results suggest that inflammatory cytokines IL-8, IL-1, IL-17 and TNF- $\alpha$ and the C and CC chemokines downstream may be used as prognostic tools for patients following percutaneous nephrolithotripsy. The findings also suggest that plasma IL-6, IL-10 and CXC chemokine upregulation is positively correlated with calcium oxolate for patients received percutaneous nephrolithotripsy. Regression analysis demonstrated that plasma concentration levels of C chemokine XCL1, CC chemokine CCL2 and TNF- $\alpha$ were positively correlated with the urinary concentration of calcium oxalate in patients during perioperative period following percutaneous nephrolithotripsy. However, further investigation using a large number population is required to confirm these results.

\section{References}

1. Sakhaee K, Maalouf NM and Sinnott B: Clinical review. Kidney stones 2012: Pathogenesis, diagnosis, and management. J Clin Endocrinol Metab 97: 1847-1860, 2012.

2. Mehmet RM, Rustu YF, Hanefi B, Mursel D, Fusun A and Mehmet I: Direct pelvic access percutaneous nephrolithotomy in management of ectopic kidney stone: A case report and literature review. Ren Fail 35: 1440-1444, 2013.

3. Goldfarb DS: ACP Journal Club. Review: Thiazide, citrate, or allopurinol reduces recurrence after $\geq 2$ kidney stone episodes. Ann Intern Med 159: JC12, 2013.

4. Heaney RP: Calcium supplementation and incident kidney stone risk: A systematic review. J Am Coll Nutr 27: 519-527, 2008.

5. Ganpule AP, Vijayakumar M, Malpani A and Desai MR: Percutaneous nephrolithotomy (PCNL) a critical review. Int J Surg 36: 660-664, 2016.

6. Jones P, Aboumarzouk OM, Rai BP and Somani BK: Percutaneous nephrolithotomy for stones in solitary kidney: Evidence from a systematic review. Urology 103: 12-18, 2017.
7. Sohail N, Albodour A and Abdelrahman K: Laparoscopic assisted transmesocolonic percutaneous nephrolithotripsy in ectopic Iliac Kidney. Urol Case Rep 7: 48-50, 2016.

8. Purkait B, Kumar M, Sokhal AK, Bansal A, Sankhwar SN and Gupta AK: Percutaneous nephrolithotomy of bilateral staghorn renal calculi in pediatric patients: 12 years experience in a tertiary care centre. Urolithiasis 45: 393-399, 2017.

9. Chatterjee M, Rath D and Gawaz M: Role of chemokine receptors CXCR4 and CXCR7 for platelet function. Biochem Soc Trans 43: 720-726, 2015.

10. Sahingur SE and Yeudall WA: Chemokine function in periodontal disease and oral cavity cancer. Front Immunol 6: 214, 2015.

11. Cerri C, Genovesi S, Allegra M, Pistillo F, Püntener U, Guglielmotti A, Perry VH, Bozzi Y and Caleo M: The chemokine CCL2 mediates the Seizure-enhancing effects of systemic inflammation. J Neurosci 36: 3777-3788, 2016.

12. McNeill E, Iqbal AJ, White GE, Patel J, Greaves DR and Channon KM: Hydrodynamic gene delivery of CC chemokine binding $\mathrm{Fc}$ fusion proteins to target acute vascular inflammation in vivo. Sci Rep 5: 17404, 2015.

13. Akagi A, Kitoh A, Moniaga CS, Fujimoto A, Fujikawa H, Shimomura Y, Miyachi Y and Kabashima K: Case of Netherton syndrome with an elevated serum thymus and activation-regulated chemokine level. J Dermatol 40: 752-753, 2013.

14. Plones T, Krohn A, Burger M, Veelken H, Passlick B, Müller-Quernheim J and Zissel G: Serum level of CC-chemokine ligand 18 is increased in patients with non-small-cell lung cancer and correlates with survival time in adenocarcinomas. PLoS One 7: e41746, 2012.

15. Costa Carvalho JL, de Brito AA, de Oliveira AP, de Castro Faria Neto HC, Pereira TM, de Carvalho RA, Anatriello E and Aimbire F: The chemokines secretion and the oxidative stress are targets of low-level laser therapy in allergic lung inflammation. J Biophotonics 9: 1208-1221, 2016.

16. Martola L, Elinder CG and Stenvinkel P: Why do patients with kidney diseases end up with a heart of stone? Disturbances in calcium-phosphate balance and chronic inflammation important causes. Lakartidningen 100: 4180-4183, 2003 (In Swedish).

17. Ramaraju K, Paranjothi AK, Namperumalsamy DB and Chennakrishnan I: Predictors of systemic inflammatory response syndrome following percutaneous nephrolithotomy. Urol Ann 8: 449-453, 2016.

18. Lu X, Gao B, Wang Y, Liu Z, Yasui T, Liu P, Liu J, Emmanuel N, Zhu Q and Xiao C: Renal tubular epithelial cell injury, apoptosis and inflammation are involved in melamine-related kidney stone formation. Urol Res 40: 717-723, 2012.

19. Mulay SR, Evan A and Anders HJ: Molecular mechanisms of crystal-related kidney inflammation and injury. Implications for cholesterol embolism, crystalline nephropathies and kidney stone disease. Nephrol Dial Transplant 29: 507-514, 2014.

20. Li W, Ma Y, Yang Q, Pan Y and Meng Q: Moist exposed burn ointment for treating pressure ulcers: A multicenter randomized controlled trial. Medicine (Baltimore) 96: e7582, 2017.

21. Hayes AF and Rockwood NJ: Regression-based statistical mediation and moderation analysis in clinical research: Observations, recommendations, and implementation. Behav Res Ther 98: 39-57, 2017.

22. Zengin K, Sener NC, Bas O, Nalbant I and Alisir I: Comparison of pneumatic, ultrasonic and combination lithotripters in percutaneous nephrolithotripsy. Int Braz J Urol 40: 650-655, 2014.

23. Naitoh Y, Yamada Y, Fujihara A, Naya Y, Hongo F, Kamoi K, Okihara K and Miki T: Percutaneous nephrolithotripsy and antegrade ureterolithotripsy in a 10-month-old infant with urinary stones in both kidney and ureter. Int J Urol 22: 128-130, 2015.

24. Hyams ES, Matlaga BR and Korley FK: Practice patterns in the emergency care of kidney stone patients: An analysis of the National hospital ambulatory medical care survey (NHAMCS). Can J Urol 19: 6351-6359, 2012.

25. Hribova P, Lacha J, Kotsch K, Volk HD, Brabcova I, Skibova J, Vitko $\mathrm{S}$ and Viklicky O: Intrarenal cytokine and chemokine gene expression and kidney graft outcome. Kidney Blood Press Res 30: 273-282, 2007.

26. Wang J, Zou H, Li Q, Wang Y and Xu Q: The expression of monocyte chemoattractant protein-1 and $\mathrm{C}-\mathrm{C}$ chemokine receptor 2 in post-kidney transplant patients and the influence of simvastatin treatment. Clin Chim Acta 373: 44-48, 2006. 
27. Tsunoda K, Shimajiri Y, Morita S, Furuta M, Kadoya Y, Yamada S, Nanjo K and Sanke T: Chronic kidney disease has a more powerful impact on peripheral arterial disease than metabolic syndrome in Japanese type 2 diabetic patients. Metab Syndr Relat Disord 7: 323-326, 2009.

28. Nevins AM, Subramanian A, Tapia JL, Delgado DP, Tyler RC, Jensen DR, Ouellette AJ and Volkman BF: A requirement for metamorphic interconversion in the antimicrobial activity of chemokine XCL1. Biochemistry 55: 3784-3793, 2016.

29. Gombert M, Dieu-Nosjean MC, Winterberg F, Bünemann E, Kubitza RC, Da Cunha L, Haahtela A, Lehtimäki S, Müller A, Rieker J, et al: CCL1-CCR8 interactions: An axis mediating the recruitment of $\mathrm{T}$ cells and Langerhans-type dendritic cells to sites of atopic skin inflammation. J Immunol 174: 5082-5091, 2005.

30. Wang XM, Hamza M, Wu TX and Dionne RA: Upregulation of IL-6, IL-8 and CCL2 gene expression after acute inflammation: Correlation to clinical pain. Pain 142: 275-283, 2009.

31. Frackowiak A, Skibiński P, Gaweł W, Zaczyńska E, Czarny A and Gancarz R: Synthesis of glycoside derivatives of hydroxyanthraquinone with ability to dissolve and inhibit formation of crystals of calcium oxalate. Potential compounds in kidney stone therapy. Eur J Med Chem 45: 1001-1007, 2010.

32. Mente A, Honey RJ, McLaughlin JM, Bull SB and Logan AG: High urinary calcium excretion and genetic susceptibility to hypertension and kidney stone disease. J Am Soc Nephrol 17: 2567-2575, 2006.

33. Zammit AR, Katz MJ, Derby C, Bitzer M and Lipton RB: Chronic Kidney disease in Non-diabetic older adults: Associated roles of the metabolic syndrome, inflammation, and insulin resistance. PLoS One 10: e0139369, 2015.

34. Budak D, Yilmaz VT, Akbas H, Suleymanlar G and Yucel G: Association between graft function and serum TNF- $\alpha$, TNFR1 and TNFR2 levels in patients with kidney transplantation. Ren Fail 37: 871-876, 2015.
35. Vazquez-Huerta DI, Alvarez-Rodriguez BA, Topete-Reyes JF, Muñoz-Valle JF, Parra-Michel R, Fuentes-Ramírez F, Salazar-López MA, Valle Y, Reyes-Castillo Z, Cruz-González A, et al: Tumor necrosis factor alpha-238 G/A and $-308 \mathrm{G} / \mathrm{A}$ polymorphisms and soluble TNF- $\alpha$ levels in chronic kidney disease: Correlation with clinical variables. Int J Clin Exp Med 7: 2111-2119, 2014.

36. Kir HM, Eraldemir C, Dervisoglu E, Caglayan C and Kalender B: Effects of chronic kidney disease and type of dialysis on serum levels of adiponectin, TNF-alpha and high sensitive C-reactive protein. Clin Lab 58: 495-500, 2012.

37. Zhang JD, Patel MB, Griffiths R, Dolber PC, Ruiz P, Sparks MA, Stegbauer J, Jin H, Gomez JA, Buckley AF, et al: Type 1 angiotensin receptors on macrophages ameliorate IL-1 receptor-mediated kidney fibrosis. J Clin Invest 124: 2198-2203, 2014.

38. Sirota JC, Walcher A, Faubel S, Jani A, McFann K, Devarajan P, Davis CL and Edelstein CL: Urine IL-18, NGAL, IL-8 and serum IL-8 are biomarkers of acute kidney injury following liver transplantation. BMC Nephrol 14: 17, 2013.

39. Vasanthakumar R, Mohan V, Anand G, Deepa M, Babu S and Aravindhan V: Serum IL-9, IL-17, and TGF- $\beta$ levels in subjects with diabetic kidney disease (CURES-134). Cytokine 72: 109-112, 2015.

40. Ahuja N, Andres-Hernando A, Altmann C, Bhargava R, Bacalja J, Webb RG, He Z, Edelstein CL and Faubel S: Circulating IL-6 mediates lung injury via CXCL1 production after acute kidney injury in mice. Am J Physiol Renal Physiol 303: F864-F872, 2012 .

41. Sinuani I, Beberashvili I, Averbukh Z and Sandbank J: Role of IL-10 in the progression of kidney disease. World J Transplant 3: 91-98, 2013

42. Kang DH, Rice M, Park NJ, Turner-Henson A and Downs C: Stress and inflammation: A biobehavioral approach for nursing research. West J Nurs Res 32: 730-760, 2010. 